\title{
Detection of Cataract and Conjunctivitis Disease Using Histogram of Oriented Gradient
}

\author{
Mrunalini Manchalwar ${ }^{\# 1}$, Krishna Warhade ${ }^{* 2}$ \\ \# ME student at Department of Electronics \& Telecommunication, \\ MIT College of Engineering, Pune, India \\ ${ }^{1}$ manchalwarm@gmail.com \\ * Professor at Department of Electronics \& Telecommunication, \\ MIT College of Engineering, Pune, India \\ ${ }^{2}$ krishna.warhade@mitcoe.edu.in
}

\begin{abstract}
The eye is a standout amongst the most critical tangible organs in the human body which comprise of pupil, iris and sclera. Eye disease is a typical medical problem around the globe. Considering the most widely recognized eye illnesses like cataract, conjunctivitis. Cataract causes a kind of blurring on the focal point prompting to diminished vision and if kept untreated for long prompts to lasting visual impairment. Conjunctivitis or pink eye is a condition where the conjunctiva of the eye is aggravated by an infection or by an allergic reaction. There are different automatic cataract, conjunctivitis recognition and order strategies accessible today. In this paper, preprocessing is done for specified eye disease images which is followed by feature extraction and classification. The image is classified as cataract disease, conjunctivitis disease and normal eye using minimum distance classifier.
\end{abstract}

Keyword-Cataract, Conjunctivitis, HOG, Minimum distance classifier.

\section{INTRODUCTION}

Biomedical image processing is an emerging research area. People can be influenced with an assortment of sicknesses and there is the need of identifying those ailments effectively. Sensitive organs like eyes are generally affected by cataract and conjunctivitis disease. The essential parts of an eye which are use to find out eye diseases are iris, pupil, sclera and are shown in Fig. 1.

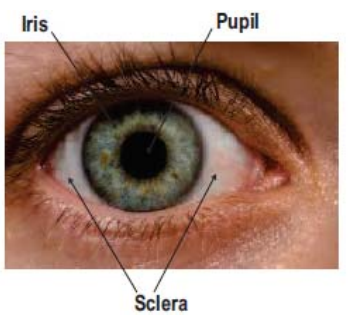

Fig. 1. Parts of an eye

\section{A. Cataract}

Cataract causes deposition of bunches of protein or a yellow-brown colour pigment decreasing the transmission of light to the back of the eye. The side effects identified with cataract incorporate clouded and blurred vision, trouble with vision during the evening and affectability with light and glare. The shadiness part of the cataract is noticeable inside the pupil and may unite out into the iris [1] [2]. Contingent upon the zone which cataract creates, it can be arranged into three: Nuclear cataract, cortical cataract and Posterior Sub Capsular (PSC) cataract. Nuclear cataract is the most widely recognized sort of cataract. It frame middle of the focal point and cause the core, or the inside, to wind up distinctly yellow or brown. Cortical cataract is wedgeshapes and form around the edges of the nucleus. (PSC) cataract form faster than the other two types and affect the back of the lens. Fig. 2 shows the normal eye and cataract contaminated eye.

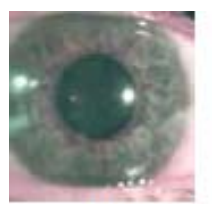

(a)

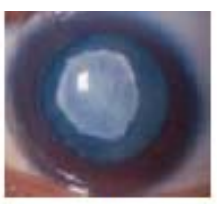

(b)

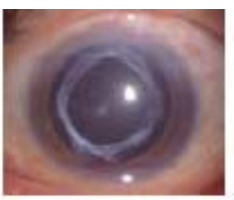

(c)

Fig. 2. (a) Normal (b) Cataract (c) Post-Cataract [7] 


\section{B. Conjunctivitis}

Conjunctivitis has side effects including pink or red shading in the uncovered white zone of the eye (Sclera), tearing and swelling of conjunctiva. Conjunctivitis can be grouped into three sorts allergic, bacterial and viral. Fig.3 (a), (b), (c) shows different conjunctivitis images and 3 (d) shows the normal eye image. Allergic conjunctivitis is the aggravation because of allergy. Bacterial conjunctivitis causes redness, swelling of the eyelid and mucopurulent release. Viral conjunctivitis might be a symptom of a contamination of the upper respiratory tract, normal cool, and sore throat.

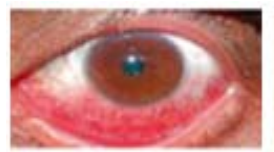

(a)

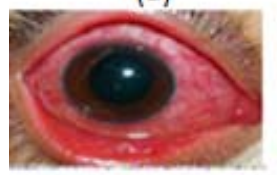

(c)

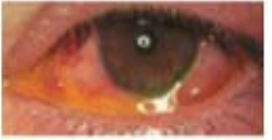

(b)

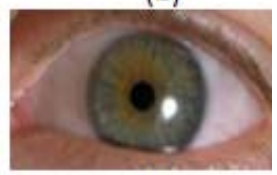

(d)

Fig.3. (a) Allergic (b) Bacterial (c) Viral conjunctivitis (d) Normal eye [6]

To discover cortical and PSC cataract, the retro illumination pictures are utilized. Nuclear cataract detection using slit lamp images [2]. A large portion of the matured individuals experience the ill effects of any of these three sorts of cataract. Optometrists ask the patients to experience different preparatory tests for these ailment discoveries, for example, reading an eye diagram, slit light examination what's more, eyes dilation. Different specialists utilize neural system [3], region based approach [4], support vector machine [5] and wavelet transform [7] [13] as a classifier for perceiving the eye sicknesses.

\section{RELATED WORK}

In this Section some of the similar work that has been done in the field of image processing for eye disease detection using different classifier has been discussed.

Meimei Yang et al. [3] proposed a system to utilize a neural network classifier for automatic cataract detection based on the classification of retinal images. The classifier building system incorporates three sections: pre-processing, highlight extraction, and classifier development. In the pre-processing section, a top-bottom hat transformation is proposed to upgrade the contrast between foreground and the object, and a trilateral filter is utilized to diminish the noise in the image. As indicated by the examination of pre-processed image, the luminance and surface message of the image are separated as classification features. The classifier is built by back propagation (BP) neural network which has two layers. In view of the clearness level of the retinal image, the patients' cataracts are ordered into typical, mild, medium or extreme ones.

H. Li et al. [4] recommended a computerized system for cataract grading. The proposed approach for nuclear cataract comprises of extraction of feature and expectation of grade. Circle estimation is finished by putting the lens utilizing thresholding, vertical and flat profile grouping. The shape of lens is then acquired by applying active shape model which is said utilizing twenty-four landmark points. Principal Component Analysis (PCA) is done on preparing tests to get the mathematical clarification of the shape and its varieties. Active Shape Model (ASM) conspire used to include the shape model into new picture subsequently decide state of the lens. SVM regression trains evaluating model and it will evaluate the grade of the image. The automatic grouping technique for cortical cataract is proposed on retro-illumination pictures. Region Of Interest (ROI) is acquired utilizing canny edge location and laplacian edge detection. Post pre-processing is done to remove noises utilizing spatial and measure channels. The cortical opacities in the got ROI is identified utilizing spoke like features. Grade is decided after the computation of aggregate area of cortical opacities.

Joydeep Tamuli et al. [5] composed a automatic image processing based technique to distinguish conjunctivitis tainted eye from an ordinary eye and characterize it as indicated by its types that is either allergic, bacterial or viral conjunctivitis. Some first and second order statistical and texture features were utilized and afterward taken after by PCA for extraction of discriminative elements since it requires the utilization of numerous scientific and statistical procedures, for example, implies, standard deviation, variance, covariance, and eigenvalues, prompting to a feature vector to look at pictures and after that ordered utilizing supervised learning technique, for example, multi-class SVM and KNN. The intensity of the contaminated eyes was additionally computed utilizing the critical red plane as indicated by its sort.

Jyoti Patil et al. [6] presented an innovation in which DIP is used for manual judgment by specialists to improve and quick outcome. The analysis of "Power of redness of pink Eye "i.e. "Conjunctivitis"; by distinguishing the region of infection in the corneal images. The rate of redness is assessed with the help of 
global thresholding. In this way average value was ascertained utilizing least and greatest value of number of pixels, the intensity of an object is $\mathrm{f}(\mathrm{x}, \mathrm{y})$ and histogram is plotted. After that the image is distinguished into two non-covering classes in view of the value of threshold $\mathrm{T}$ in the histogram. If $\mathrm{f}(\mathrm{x}, \mathrm{y})<\mathrm{T}$ into a class called background information and $\mathrm{f}(\mathrm{x}, \mathrm{y})>\mathrm{T}$ into another class called object. In the segmented binary picture, background pixels have value of zero while object pixels have value 1 . When the value of threshold $T$ is constant, this is called global thresholding.

Jagadish Nayak et al. [7] utilized different picture preparing procedures to discover the components in the distinctive classes of optical eye pictures. The pre-processing operations completed are normalization and transformation of RGB colour band to intensity-hue-saturation. Normalization completed maintains a strategic distance from shading variety of eye pictures among every patient. In the intensity-shade immersion representation, the intensity is processed without changing the relative shading estimations of the pixels. Highlight extraction operations like Small Ring Area (SRA), Big Ring Area (BRA), Edge pixel check (EPC) what's more, Object Perimeter are performed. In SRA, the inner area of the cornea is gotten, which is more whitish in cataract pictures than on account of typical and post-cataract pictures. External zone of the cornea is acquired from BRA which would be brighter in shading in cataract pictures. Canny edge detection is utilized to discover the edges. It will give clear edge identification indeed, even in the noise state by utilizing thresholding strategy EPC checks the quantity of white pixels in the distinguished edge.

Melih Gunay et al. [9] built up an automated framework for recognition of adenoviral conjunctivitis. In such a framework they arranged a basic setup in which camera was introduced outside the specialist's office that takes patients confront pictures for potential danger of conveying Ad-Cs. This innovation incorporates the acknowledgment of face, choice of eyes and extraction of eye sclera using automated GrabCut algorithm in a pipeline design. After that System distinguished eye is normal or infected. They accomplished 93\% precision in disease detection.

Anju J. S and Anju S. L [10] proposed a new technique for vessel detection in sclera by consolidating the idea of frangi filter and wavelet transform. Sclera vein detection is an important step in human recognizable proof. Sclera is the white and hazy external defensive covering of an eye. Sclera area detection is utilized to distinguish the relevant portion of the sclera from the eye area for further preparing and distinguishing proof. Sclera range is distinguished in view of Otsu's thresholding strategy. Vein structure in the sclera area portion is recognized based upon frangi filter and wavelet transform. In Frangi filter the Eigen value examination of the hessian matrix is performed. At that point wavelet decomposition of the filtered picture is utilized to acquire the vessel structure in the sclera.

Sclera acknowledgment can be utilized for human distinguishing proof. Be that as it may, if the sclera pictures can't be legitimately segmented by the framework or the pictures of sclera examples are defocused or potentially soaked, it can altogether influence the exactness of sclera acknowledgment. Zhi Zhou et al. [11] proposed a comprehensive sclera picture quality measure which can rapidly distinguish if the picture has a substantial eye, evaluate the picture quality, assess the division precision, and measure if the picture has adequate element data for acknowledgment. Likewise, it utilized Dempster Shafer Theory (DST) to combine the quality score, division score, and highlight score together to produce the general combination score. It is observationally confirmed utilizing the UBIRIS database that the proposed quality measure is exceedingly related with the execution of sclera acknowledgment.

Wenai Song et al. [12] used semi-supervised figuring out how to construct a classifier for programmed arrangement and reviewing of cataract. Using a substantial size of unlabelled illustrations together with a little piece of named cases to learn hypothesis is known as semi supervised learning. They utilized tri-preparing which creates three classifiers from the first named illustrations. At that point using unlabelled cases to refine initial classifier in an emphasize technique. Probes genuine word informational indexes included 476 marked illustrations and 4902 unlabelled cases.

Yunendah Nur Fuadah et al. [13] researched the ideal combination candidate of statistical texture features that is give most astounding exactness to cataract detection. In this examination, they utilized K-Nearest Neighbor (K-NN) as characterization technique that will be actualized on android cell phone. Result demonstrates that the ideal combinations of texture features are disparity, contrast and consistency. The most astounding exactness of the framework is $97.5 \%$.

Manpreet kaur et al. [14] exhibited a cell phone based on smart system incorporation and micro lenses are attached to mobile that permits patients in remote zones to have customary eye checks and the creative advancement of infection finding. The official portable mobile indicative system to examination of retinal pictures taken by microscopic lens to recognize retinal infection conditions. In Table I we have compared various related work with respect to algorithm used, merits and accuracy. From the literature review we find that an algorithm based on supervised learning [5] and automated Grabcut [9] provides more accuracy than other algorithms. 
TABLE I. Comparative study of different eye diseases

\begin{tabular}{|c|c|c|c|}
\hline Author & Algorithm used & Merits & Accuracy \\
\hline $\begin{array}{l}\text { Meimei } \\
\text { Yang et } \\
\text { al. [3] }\end{array}$ & $\begin{array}{l}\text { Back } \\
\text { propagation } \\
\text { neural network }\end{array}$ & $\begin{array}{l}\text { Improve diagnosis } \\
\text { efficiency of the } \\
\text { ophthalmologist and } \\
\text { reduce the physical } \\
\text { and economic burden } \\
\text { of the patients \& } \\
\text { society }\end{array}$ & $82 \%$ \\
\hline $\begin{array}{l}\text { Huiqi Li } \\
\text { et al. [4] }\end{array}$ & SVM regression & $\begin{array}{l}\text { Computer aided } \\
\text { diagnosis system } \\
\text { provides objective } \\
\text { grading of cataract }\end{array}$ & $89 \%$ \\
\hline $\begin{array}{l}\text { Joydeep } \\
\text { tamuli et } \\
\text { al. [5] }\end{array}$ & $\begin{array}{l}\text { Supervised } \\
\text { learning } \\
\text { methods multi } \\
\text { class SVM and } \\
\text { K-NN }\end{array}$ & $\begin{array}{l}\text { Method is efficient, } \\
\text { computationally } \\
\text { fast ,cost is very low }\end{array}$ & $\begin{array}{l}\text { 95\% for } \\
\text { multi class } \\
\text { SVM } \\
85 \% \text { for K- } \\
\text { NN }\end{array}$ \\
\hline $\begin{array}{l}\text { Jyoti patil } \\
\text { et al. [6] }\end{array}$ & $\begin{array}{l}\text { Global } \\
\text { thresholding }\end{array}$ & $\begin{array}{l}\text { Best method for } \\
\text { segmentation of } \\
\text { image which easily } \\
\text { calculates the } \\
\text { number of pixels }\end{array}$ & $80 \%$ \\
\hline $\begin{array}{l}\text { Jagdish } \\
\text { Nayaak } \\
{[7]}\end{array}$ & $\begin{array}{l}\text { Support vector } \\
\text { machine }\end{array}$ & $\begin{array}{l}\text { SVM classifier is } \\
\text { effective to the tune } \\
\text { of accuracy }\end{array}$ & $88 \%$ \\
\hline $\begin{array}{l}\text { Melih } \\
\text { Gunay et } \\
\text { al. [9] }\end{array}$ & $\begin{array}{l}\text { Automated } \\
\text { Grabcut } \\
\text { algorithm, RGB } \\
\text { thresholding, } \\
\text { GLCM }\end{array}$ & $\begin{array}{l}\text { This is automatic fast } \\
\text { and cost effective } \\
\text { diagnosis }\end{array}$ & $96 \%$ \\
\hline $\begin{array}{l}\text { Wenai } \\
\text { Song et } \\
\text { al. [12] }\end{array}$ & $\begin{array}{l}\text { Bayesian } \\
\text { network }\end{array}$ & $\begin{array}{l}\text { Involves function } \\
\text { estimation on } \\
\text { labelled and } \\
\text { unlabelled data }\end{array}$ & $88 \%$ \\
\hline
\end{tabular}

\section{III.PROPOSED SYSTEM}

The proposed system for detection of cataract and conjunctivitis eye disease is described in Fig.4. It consists of following process. We are acquiring image from the database and these images are preprocessed for image enhancement. Then features of the image are extracted using HOG and are classified by using minimum distance classifier.

\section{A. Image Acquisition and Preprocessing}

This is the first and the most essential step which takes digital input picture in jpeg or png format from the database. Image pre-processing typically denotes a processing step transforming a source image into a new image which is fundamentally similar to the source image, but differs in certain aspects, e.g. improved contrast. According to the above definition, pre-processing results in changing the brightness of individual image pixels. This step includes the physical transformation of the RGB and the grayscale image. 


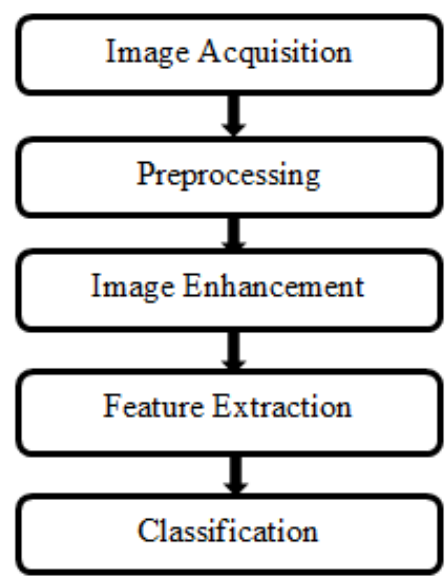

Fig. 4. Proposed system block diagram

\section{B. Image Enhancement}

Image enhancement is the process of adjusting digital images so that results are more suitable for display or further image analysis. Median filter is used to remove the noise in smooth patches or smooth regions of a signal, but adversely affect edges, and making it easier to identify key features.

\section{Feature Extraction}

This step is basically responsible for extracting the key element which serves as the basis for analysing the output. This involves basic morphological features of an eye. The Histogram of Oriented Gradient (HOG) is used to implement the method. This technique counts occurrences of gradient orientation in localized portion of an image. It is computed on a dense grid of uniformly spaced cells uses overlapping local contrast normalization for improved accuracy. Relevant features like the disease part containing the pupil, iris, sclera are extracted and their matching score values are calculated to get the result.

\section{Classification}

Classification rules are defined on the basis of feature extracted for different eye images. Once the features have been extracted, we continue advance by grouping the pictures and distinguishing the disease. We have used minimum distance classifier to classify cataract, conjunctivitis eye infection and normal eye.

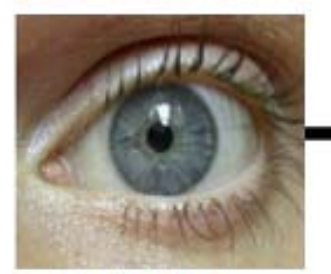

Acquired Image

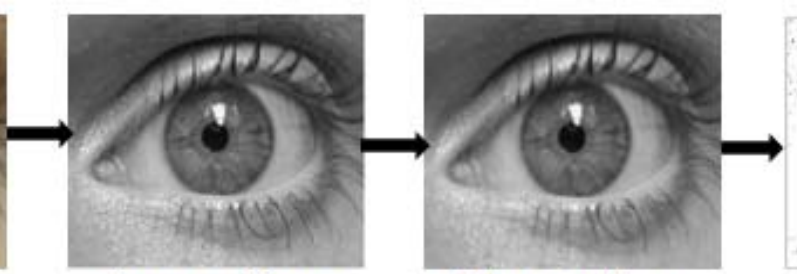

Preprocessed image
Enhancement image

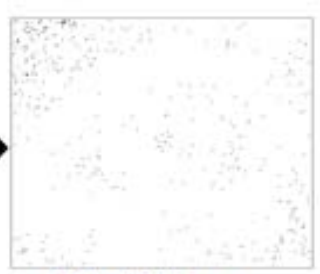

Extracted image

Fig. 5.The proposed system flow

The proposed system flow is described in Fig. 5. The flow is shown for one sample image which is acquired from database and input image is preprocessed by converting RGB to grey. After that we get the enhanced image using median filter. Then HOG is used for collecting extracted image.

\section{IV.EXPERIMENTAL RESULT}

We have tested the images for cataract and conjunctivitis disease by using our algorithm. Fig. 6 shows the result on sample of two cataract images, two conjunctivitis images and two normal eye images. All images to the left hand side of Fig. 6 shows the input sample images, whereas right hand side images are resultant images after applying algorithm. From the result, it can be clearly observed that our algorithm automatically identifies cataract, conjunctivitis and normal eye images. 

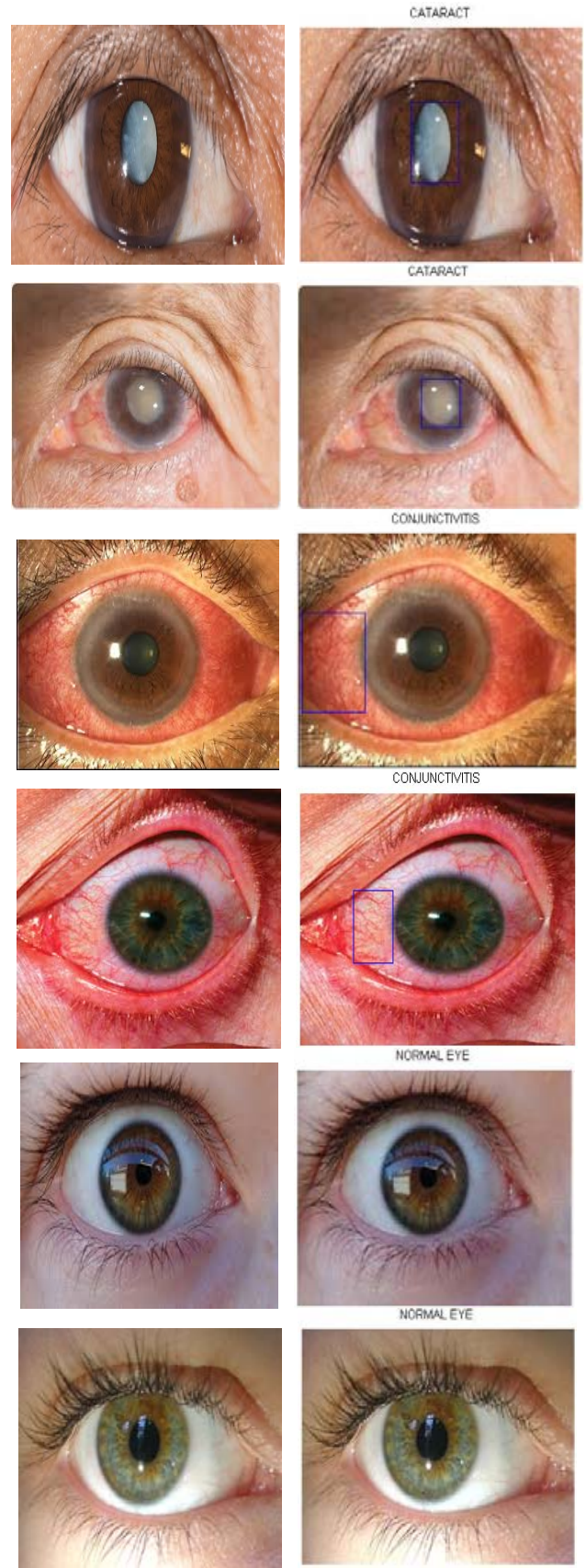

Fig.6. Input images (left side) and their resulting images (right side).

\section{Conclusion}

The proposed algorithm demonstrates the approach of cataract, conjunctivitis disease identification by extracting best components from pupil, sclera portion of eye utilizing HOG. We have used HOG for feature extraction and their extracted feature are classified using minimum distance classifier. Our algorithm clearly classifies normal eye and eye with cataract and conjunctivitis disease. In the future, we will work on identifying other eye diseases such as Stye, Subconjunctival hemorrhage, Chalazion. This will help in predicting the eye disease, before any serious ill effect on the eye.

\section{REFERENCES}

[1] A. Ektesabi, A. Kapoor, "Extract pupil and Iris boundary detection”, $2^{\text {nd }}$ International Conference on Control, Instrumentation and Automation (ICCIA), pp. 1217-1221, 2011.

[2] Amol B. Jagadale and D. V. Jadhav, "Early detection and categorization of cataract using slit-lamp images by hough circular transform”, International Conference on Communication and Signal Processing, pp. 0232-0235,April 6-8, 2016.

[3] Meimei Yang, Ji-Jiang Yang, Qinyan Zhang, Yu Niu, Jianqiang Li, "Classification of retinal image for automatic cataract detection”, IEEE $15^{\text {th }}$ International Conference on e-Health Networking, Applications and Services, pp. 674-679, 2013.

[4] Huiqi Li, JooHwee Lim, Jiang Liu, Damon Wing Kee Wong, Ngan Meng Tan, Shijian Lu, Zhuo Zhang, Tien Yin Wong, “Computerized systems for cataract grading”, $2^{\text {nd }}$ International Conference on Biomedical Engineering and Informatics, pp. 1-4, 2009. 
[5] Joydeep Tamuli, Aishwarya Jain, Aaishwarya V. Dhan, Anupama Bhan, Malay Kishore Dutta, "An image processing based method to identify and grade conjunctivitis infected eye according to its types and intensity", $8^{\text {th }}$ International Conference on Contemporary Computing (IC3), pp. 88-92, 2015

[6] Jyoti Patil, Anant. L. Chaudhari, "Intensity observation of conjunctivitis using DIP”, International Journal of Applied Information Systems (IJAIS), Foundation of Computer Science FCS, New York, USA, vol 4, no.4, October 2012.

[7] Jagadish Nayak, "Automated classification of normal, cataract and post cataract optical eye images using SVM classifier", World Congress on Engineering and Computer Science (WCECS), vol I, pp. 23-25 October, 2013.

[8] Amit Asish Bhadra, Manu Jain, Sushila Shidnal, “Automated Detection of Eye Diseases”, IEEE WiSPNET Conference, pp. 13411345, 2016.

[9] Melih Gunay, Irem Kucukoglu, Evgin Goceri, Taner Danisman, Fadi Alturjman, “Automated detection of adenoviral conjunctivitis disease from facial images using machine learning”, IEEE $14^{\text {th }}$ International Conference on Machine Learning and Applications, pp. 1204-1209, 2015.

[10] Anju J. S, Anju S. L, "An effective method for blood vessel detection in sclera”, International Journal of Engineering Research \& Technology (IJERT), vol. 3 Issue 3, March - 2014.

[11] Zhi Zhou, Eliza Y. Du, N. Luke Thomas, “A comprehensive sclera image quality measure”, $11^{\text {th }}$ International Conference on Control, Automation, Robotics and Vision Singapore, pp. 638-643, 7-10

[12] Wenai Song, Ping Wang, Xudong Zhang, Qing Wang, "Semi-supervised learning based on cataract classification and grading”, IEEE $40^{\text {th }}$ Annual Computer Software and Applications Conference, pp. 641-646, 2016

[13] Yunendah Nur Fuadah, Agung W. Setiawan, Tati L.R. Mengko, and Budiman, "Mobile cataract detection using optimal combination of statistical texture analysis", $4^{\text {th }}$ International Conference on Instrumentation, Communications, Information, and Biomedical Engineering (ICICI-BME), pp. 232-236, November 2-3, 2015.

[14] Manpreet Kaur, Jaspreet Kaur, Ravinder Kaur, "Low cost cataract detection system using smartphone” International Conference on Green Computing and Internet of Things (ICGCIoT), pp. 1607-1609, 2015.

[15] Sivapriya C, Vignesh R, "Eye disease detection using daubechies wavelet transform”, International Journal for Innovative Reasearch in science \& technology (IJIRST), vol 1, Issue 12, May 2015.

[16] Niya C P, “Automatic cataract detection and classification systems: A survey”, TechS Vidya e-Journal of Research, vol. 3 (2014-15), pp. 28-36.

[17] V. Harini, V Bhanumathi, "Automatic cataract classification system”, International Conference on Communication and Signal Processing, pp. 0815-0819, April 6-8, 2016, India.

\section{AUTHOR PROFILE}

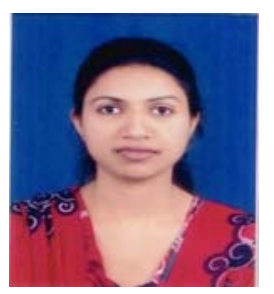

Mrunalini D. Manchalwar received the Bachelor of Engineering in Electronics in 2013 from Govindrao Wanjari College of Engineering and technology, Nagpur, India. Currently she is pursuing Master of Engineering in VLSI \& Embedded Systems from MIT College of Engineering, Pune, India. Her research interest is in the area of Image Processing, Signal Processing and biomedical signal processing.

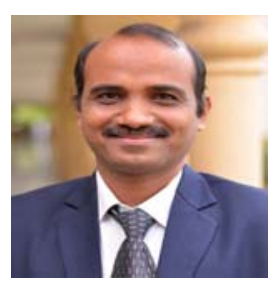

Krishna K. Warhade received the Bachelor of Engineering in Electronics in 1995 and Master of Engineering in Instrumentation in 1999 both from Shri Guru Govind Singhaji Institute of Engineering and Technology, Nanded, India and PhD in Nov 2010 from the department of Electrical Engineering, Indian Institute of Technology, Bombay, India. He has 21 years of teaching experience. He is currently working as a Dean R \& D at MIT College of Engineering, Pune, India. His research interest are in the area of Image Processing, Signal Processing, Video shot boundary detection, Wavelets and biomedical signal processing. 